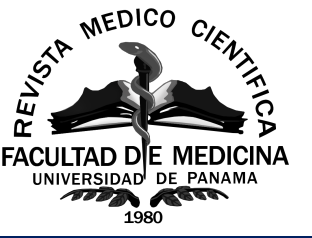

\title{
Factores de riesgo de enfermedad diarreica aguda en menores de 5 años
}

\author{
Risk factors for acute diarrheal disease in children under 5 years of age \\ Yamilka Yard Foster *; Jenia Núñez Ortega †; José Correoso Guevara ¥ \\ *Residente de Ginecología y Obstetricia. Hospital Santo Tomás, Panamá \\ †Médico General. Centro de Vacunación e Investigación (Cevaxin), Panamá \\ $\ddagger$ Médico General. Hospital Santo Tomás, Panamá
}

\section{RESUMEN}

La enfermedad diarreica aguda constituye un problema de salud pública en menores de 5 años; principalmente en países en vías de desarrollo. Cada año las diarreas causan una considerable morbilidad infantil reportando alrededor de 1700 millones de casos, representando la segunda causa de mortalidad con aproximadamente 525000 muertes cada año.

En Panamá, la enfermedad diarreica aguda se coloca dentro de las principales causas de morbimortalidad con 24.3 defunciones por cada 100000 menores de 5 años y con 94564 casos registrados para el 2017.

En contraste, en Panamá no se cuentan con estudios recientes sobre los principales factores de riesgo que impactan sobre el desarrollo de la enfermedad diarreica aguda, esto aunado al hecho que existen factores sociales que propician su aparición. El conocimiento de los factores de riesgo permitirá la identificación de medidas de prevención que disminuyan la incidencia de esta enfermedad.

\section{ABSTRACT}

Acute diarrheal disease constitutes a public health problem in children under 5 years of age; mainly in developing countries. Every year, diarrhea causes considerable infant morbidity, reporting around 1700 million cases, representing the second leading cause of mortality with approximately 525000 deaths each year.

In Panama, acute diarrheal disease is placed among the main causes of infant mortality and morbidity with 24.3 deaths for every 100000 children under 5 years of age and with 94564 cases registered for 2017.

In our country, we do not have recent studies on the main risk factors that impact on the development of acute diarrheal disease. This, coupled with the fact that there are social factors that promote its appearance, makes it vital to seek preventive measures that decrease the incidence of this disease.

\section{|}

Correspondencia: yamilkayard@hotmail.com

Recibido: $15 / 7 / 2020$

Aceptado: 23/3/2021

\section{PALABRAS CLAVE:}

Diarrea; lactancia materna; salud pública; factores de riesgo.

\section{KEY WORDS:}

Diarrhea; breastfeeding; public health; risk factors. a enfermedad diarreica aguda (EDA) es una importante causa de morbimortalidad en muchas partes del mundo, sobre todo en los países en vías de desarrollo, siendo los niños la población más vulnerable. ${ }^{[1-3]}$

La Organización Mundial de Salud (OMS) define a la enfermedad diarreica como el paso de tres o más deposiciones sueltas o líquidas por día, o con más frecuencia de lo normal para la persona; por lo general, es un síntoma de infección gastrointestinal que puede ser causado por una variedad de microorganismos. Esta infección se propaga a través del consumo de agua o alimentos contaminados, y el contacto con personas con una higiene deficiente ${ }^{[2]}$

Existen tres tipos clínicos: la acuosa aguda (dura varias horas hasta 7 a 14 días), la disentérica (aguda con sangre) y la persistente (dura 14 días o más). ${ }^{[2,3]}$ 
A pesar de que cualquiera la puede padecer, esta enfermedad está ligada fuertemente al nivel socioeconómico y cultural, siendo más susceptibles las personas de bajos recursos económicos, inmunocomprometidos y malnutridos. Esto se debe a que el $80-90 \%$ de los casos son asociados a condiciones ambientales desfavorables entre ellas: saneamiento inadecuado y presencia de agua contaminada. ${ }^{[1-4]}$

En los países en desarrollo, la salud infantil se ve afectada por la situación socioeconómica de los padres, constituyendo el entorno inmediato para el niño; situación que demuestran Ayele et al. con una influencia significativa entre los ingresos familiares y la prevalencia de diarrea. ${ }^{[4]}$

Los niños menores de 5 años pueden tener cuadros de diarrea aguda hasta 3 veces al año; durante estos episodios, el niño queda privado de los nutrientes necesarios para su desarrollo. Si no se da la reposición de éstos durante la fase de convalecencia, se genera un déficit que afectará su crecimiento. Por esto se considera una causa importante de malnutrición y retraso pondoestatural. ${ }^{[2]}$

\section{EPIDEMIOLOGÍA}

Según la OMS y los estudios revisados, esta enfermedad causa aproximadamente 525000 muertes al año, posicionándose entre la cuarta y quinta causa de muerte en niños menores de 5 años. ${ }^{[1,2]}$

En Panamá, la enfermedad diarreica aguda se posiciona entre las primeras causas de muerte en los infantes. Según los indicadores básicos de salud del Ministerio de Salud (MINSA), en el 2014 ocurrieron 18.3 defunciones y en el 201724.3 defunciones por cada 100000 niños menores de 5 años con 94564 episodios diarreicos reportados en el boletín estadístico del MINSA en el 2017. ${ }^{[5-7]}$
La última encuesta de indicadores múltiples por conglomerados de la contraloría general de la república en el 2013 informó que el porcentaje de niños menores de 5 años que presentaron casos de diarrea fue del $12,1 \%$ y de éstos sólo el $52.7 \%$ recibió tratamiento con las sales de rehidratación oral y alimentación continuada. ${ }^{[8]}$

\section{FACTORES DE RIESGO}

\section{Vivienda}

Es importante conocer el tipo de vivienda en que reside el menor de edad y la cantidad de habitantes por habitación dentro de la misma. El estudio de Sinmegn et al. mostró que el $94.7 \%$ de 925 pacientes residían en hogares con suelo de tierra. ${ }^{[9]}$ Mamo, Hailu A., en su estudio realizado en Etiopía, demostró asociación estadística entre vivir en casas con ganado y las enfermedades diarreicas infantiles, además, que los niños que residían en zonas rurales tenían aproximadamente dos veces más probabilidades de tener enfermedades diarreicas que los niños que vivían en las zonas urbanas [OR: 2.527, IC 95\%: (1.847 $-7.538)]$. $^{[7]}$

En cuanto al tamaño familiar en el hogar, a mayor número, mayor riesgo de desarrollar EDA. Según Godana y Mengistie, se presentó un mayor riesgo en aquellos pacientes con EDA que residían junto a más de 6 habitantes. ${ }^{[10]}$

Herrera et al., en su estudio realizado en Bolivia, encontraron al hacinamiento como factor de riesgo para el desarrollo de EDA con deshidratación severa con un odds ratio (OR) 3.92 e intervalo de confianza (IC) 1.58-9.83.[11] Resultados similares fueron encontrados en el estudio de Cáceres et al. donde el hacinamiento resultó factor de riesgo para la enfermedad diarreica aguda con diferencia estadísticamente significativa $(p<0.05) .{ }^{[7]}$ 


\section{Nivel Socioeconómico}

Los niños pertenecientes a hogares con bajo estrato socioeconómico fueron significativamente asociados con el desarrollo de diarrea infantil (odds ratio: 7.14; intervalo de confianza: 2.19-23.32). ${ }^{[12]}$ Equiparable al estudio de Godana Y Mengistie donde el ingreso familiar (OR: 5.89, IC 3.97-8.77) presentó significancia estadística. ${ }^{[10]}$

\section{Agua Potable y Disposición de Excretas}

Ligado al hogar está el servicio de agua potable y la deposición de excretas, ya sea baño higiénico u otros, que condicionan la higiene del hogar y del niño. Se sabe que el agua potable es un factor muy importante en el desarrollo de enfermedad diarreica porque es parte vital de la vida humana, ya sea al consumirla en la alimentación o utilizarla en las necesidades básicas. Un ejemplo de esto es el resultado obtenido en el estudio de Godana y Mengisitie en Derashe, Etiopía, que mostró como factor de riesgo de EDA no contar con agua potable, al igual que no tratar el agua mediante ebullición, filtración o productos químicos. En este mismo estudio se evidenció el riesgo elevado de diarrea, tanto en adultos como infantes, en aquellos que no contaban con una adecuada deposición de excretas. ${ }^{[10]}$

La falta de saneamiento y el suministro de agua contaminada constituyen los principales factores desencadenantes de la alta morbimortalidad de la EDA, con la muerte de un niño menor de cinco años cada 15 segundos. ${ }^{[10]}$

\section{Lactancia Materna Exclusiva}

En cuanto a la alimentación del niño, está comprobado que la lactancia materna exclusiva, como mínimo hasta los primeros 4 meses de edad, es la mejor alimentación que puede tener el niño, y que suple todos los requerimientos a esta edad, incluyendo inmunidad. Huffman et al., en un estudio realizado en Brasil, demostró que los bebés que no son amamantados tienen 25 veces más riesgo de morir de diarrea que aquellos que reciben lactancia materna exclusiva. ${ }^{[13]}$

Cáceres et al. en su estudio demostró como factor de riesgo una lactancia materna menor de 3 meses. ${ }^{[7]}$ Lo mismo fue demostrado por Mariños-Anticona et al. en un estudio realizado en Perú hacia el año 2014 en el que resultó como factor protector de desarrollar diarrea, la lactancia materna exclusiva hasta los 6 meses de edad. ${ }^{[14]}$

La lactancia materna exclusiva protege al niño de enfermedades al disminuir el riesgo de exposición a bacterias entéricas presentes en agua y comida contaminada. ${ }^{[15]}$ En el metanálisis de Lamberti et al. se encontró evidencia de los efectos protectores de la lactancia materna contra la incidencia de diarrea, su prevalencia y las hospitalizaciones relacionadas con la misma. Sus resultados destacaron la importancia de la lactancia materna para proteger contra la morbilidad y mortalidad específica de la diarrea durante los primeros 2 años de vida. ${ }^{[16]}$

\section{Estado Nutricional}

La vigilancia del estado nutricional es un aspecto importante. Según la OMS, la malnutrición es una de las principales causas del desarrollo de enfermedad diarreica, siendo los niños que están desnutridos o con deficiencias de inmunidad, los que presentan mayor riesgo. ${ }^{[17]}$

En el estudio realizado por Herrera et al. para el año 2017 en Bolivia, se encontró que los pacientes con mayor grado de desnutrición presentaron un mayor riesgo de diarrea con deshidratación y diarrea persistente, además de un mayor riesgo de complicaciones secundarias. ${ }^{[11]}$

El metanálisis de Ganguly et al. del 2016 establece la asociación significativa de la enfermedad diarreica con la desnutrición infantil OR 1.71, con un IC (1.29 $2.28)$, permitiendo concluir que la intensificación de

www.revistamedicocientifica.org 
medidas para el control de la desnutrición podría ayudar a la disminución de la enfermedad. ${ }^{[12]}$

Lo mismo fue corroborado en el Estudio Global Entérico Multicéntrico realizado en Kenia, África, donde se encontró que los niños con mayor grado de malnutrición (altura para la edad Z score < -2) presentaron episodios de diarrea más prolongados. $^{[18]}$

\section{Anemia}

Según el metanálisis de Ganguly et al. del 2016, la anemia está asociada como factor de riesgo significativo para contraer infecciones en los primeros años de vida incluyendo EDA, situación que también se ve reflejada en un estudio peruano del 2012 que informa que aquellos niños menores de 3 años con anemia moderada presentaron un OR de 3,09 e IC $(1,30-7,37)$ de presentar un episodio diarreico agudo. A la luz de estos hallazgos, se puede concluir que controlar la anemia en los niños puede reducir la morbilidad y la mortalidad resultante debido a la diarrea severa. Este puede ser un enfoque novedoso para la prevención de la diarrea, ya que la mayoría de las intervenciones disponibles están dirigidas a medidas de saneamiento y agua potable. ${ }^{[12,14]}$

\section{Zinc}

El papel del zinc en la prevención de la diarrea ha sido señalado por el estudio de Mazunder et al. sobre impartir educación a los cuidadores sobre la administración de suplementos de zinc, que reporta un efecto protector significativo con un OR 0.34 $(0.18-0.63) .^{[12]}$

La deficiencia de zinc se asocia con un mayor riesgo de infecciones gastrointestinales, efectos adversos en la estructura y función del tracto gastrointestinal y una función inmune deteriorada. El zinc suplementado beneficia a los niños con diarrea porque es un micronutriente vital esencial para la síntesis de proteínas, el crecimiento y la diferenciación celular, la función inmune y el transporte intestinal de agua y electrolitos. ${ }^{[19,20]}$

\section{Cuidador}

Por último, el cuidador del niño, usualmente la madre, es quien tiene el control de algunos de los factores de riesgo de EDA, como lo es la lactancia, el tratamiento del agua, preparación de alimentos, deposición de excretas, entre otros. Se ha visto que el riesgo de tener una EDA es casi el doble en los niños que reciben los cuidados de una madre joven (menor de 20 años) con una baja educación en salud en comparación con aquellos niños cuyas madres no son jóvenes. ${ }^{[9,21]}$

PREVENCIÓN

Un pilar para la prevención de la enfermedad diarreica aguda está constituido por la lactancia materna. En Panamá, según estudios realizados por el Fondo de las Naciones Unidas para la Infancia (UNICEF), la tasa de lactancia materna exclusiva los primeros seis meses está alrededor del 25\%, un porcentaje que se encuentra muy por debajo de la media mundial. ${ }^{[20]}$

Un estudio de costo beneficio realizado por el Instituto Conmemorativo Gorgas de Estudios para la salud (ICGES) para el año 2006 reveló que el 30,6\% de las madres daban lactancia materna exclusiva durante el primer mes, pero solamente un $6.8 \%$ continuaba con la lactancia materna exclusiva hasta el sexto mes. ${ }^{[22]}$

Por su parte, la Encuesta Nacional de Salud Sexual y Reproductiva (ENASSER 2009) reveló que la lactancia materna exclusiva en áreas urbanas (17.7\%) es inferior, comparada con las áreas rurales (38.3\%) e indígenas (52.0\%) del país. ${ }^{\text {[23] }}$ 
Hay que tener en cuenta que existen múltiples factores que pueden influir sobre el inicio y duración de la lactancia materna, ya sean económicos, sociales, laborales o sanitarias. Un aspecto que ha sido estudiado en múltiples ocasiones es el impacto de las recomendaciones médico-sanitarias. ${ }^{[19]}$

En el estudio previamente mencionado, ENASSER 2009 , se encontró que solo un $49.4 \%$ de las pacientes entrevistadas habían recibido información sobre la lactancia materna en los centros de salud. ${ }^{[23]}$

En un estudio de conocimientos, actitudes y prácticas en personal de salud en unidades de atención primaria realizado por el ICGES en colaboración con el MINSA y la Caja de Seguro Social para el 2011, se reveló que en términos generales el personal obtuvo un porcentaje de respuestas acertadas del $54.7 \%$, desglosado en conocimientos $35.1 \%$ de respuestas acertadas, actitudes $81.6 \%$ y prácticas $58.0 \%$, encontrándose mejores resultados en el personal con mayor grado educativo. A pesar de que este estudio fue realizado sólo en unidades de la región metropolitana y San Miguelito, demuestra la necesidad de mantener y fortalecer los conocimientos y prácticas sobre la lactancia materna en el personal de salud de todo el país, además de mejorar las estrategias de promoción y prevención. ${ }^{[19]}$

Otra de las medidas adoptadas para controlar el problema la constituye la vacuna contra rotavirus. De acuerdo con datos de la Organización Panamericana de la Salud, esta vacuna fue aprobada e introducida al Programa Ampliado de Inmunización (PAI) de Panamá el 14 de marzo de 2006. Se trata de Rotarix ${ }^{\circledR}$, una vacuna oral monovalente de virus vivo atenuado con una cepa G1P que expresa las proteínas estructurales VP4 y VP7 de la mayoría de los serotipos de rotavirus humanos. La misma se aplica de acuerdo a las últimas normas del PAl a niños entre
2 a 8 meses, con dos dosis que deben ser aplicadas con un intervalo entre 4 y 8 semanas. ${ }^{[24,25]}$

Según estudios de la relación costo beneficio realizados en 4025 lactantes en Panamá, la introducción de la vacuna antirotavírica podría evitar anualmente 13685 visitas médicas ambulatorias, 1140 hospitalizaciones y 24 muertes, por enfermedad diarreica aguda en los primeros 5 años de vida. ${ }^{[24]}$

TRATAMIENTO

En los infantes, la EDA suele tener un curso autolimitado, de ahí que la base del tratamiento esté dirigida hacia la reposición de los líquidos y electrolitos perdidos, además de mantener una adecuada nutrición. La principal complicación y mayor causa de mortalidad en la EDA está asociada a la deshidratación, ${ }^{[26,27]}$ por lo tanto, el tratamiento está dirigido hacia la identificación y clasificación del grado de deshidratación de acuerdo a la historia clínica y el examen físico. ${ }^{[26]}$

En términos generales, los pacientes con deshidratación leve a moderada reciben hidratación mediante sueros de rehidratación oral, mientras que la hidratación intravenosa se reserva para casos severos. ${ }^{[27]}$

En cuanto a la nutrición, se recomienda iniciar la dieta luego de tres o cuatro horas de rehidratación, ya sea oral o intravenosa, utilizando la dieta habitual del paciente, y evitando introducir alimentos nuevos durante el periodo de enfermedad. En los casos más leves de la enfermedad, no se debe suspender nunca la dieta, además que, en aquellos infantes que son amamantados, no se recomienda la suspensión de la lactancia materna, excluyendo únicamente a los pacientes con casos de diarrea e intolerancia a la lactosa, a los que se le debe ofrecer fórmulas sin lactosa o productos libres de lácteos fermentados si el paciente es mayor de un año. ${ }^{[26,27]}$

www.revistamedicocientifica.org 
En este grupo etario, los principales agentes etiológicos son agentes virales e incluso los agentes bacterianos involucrados, por lo general, tienen un curso autolimitado, por lo que no está recomendado el uso rutinario de antibióticos. No existe un consenso absoluto para la indicación de antimicrobianos. En términos generales, se pueden iniciar en aquellos pacientes en los cuales se sospecha de sepsis bacteriana con diseminación gastrointestinal o aquellos con compromiso sistémico severo. ${ }^{[27]}$

Otras terapias que pueden ser utilizadas incluyen: probióticos, racecadotril y diosmectita, los cuales tienen un efecto favorable en el curso clínico de la enfermedad. Los probióticos y su combinación con prebióticos (simbióticos) contribuyen esencialmente a aliviar y acortar el curso de la enfermedad, mientras que el racecadotril y la diosmectita disminuyen la pérdida de agua fecal y electrolitos. El uso de loperamida y agentes antieméticos (Ej. Metoclopramida) no está recomendado en niños por el gran espectro de efectos adversos. ${ }^{[27]}$

La OMS, además, recomienda el uso rutinario de Zinc en los pacientes con enfermedad diarreica aguda, a una dosis de 20 miligramos por día para niños mayores de seis meses o $10 \mathrm{mg}$ por día en niños menores de seis meses durante 10 a 14 días, principalmente en los países en vías de desarrollo donde existe, por lo general, mayor deficiencia de este nutriente. ${ }^{[19,28]}$

Además, se ha demostrado que la suplementación con zinc reduce la proporción de los casos de diarrea prolongada y la necesidad de hospitalización, por lo que está incluido dentro de las recomendaciones del MINSA. ${ }^{[19,20]}$

\section{CONCLUSIÓN}

La enfermedad diarreica aguda constituye una causa importante de mortalidad en menores de 5 años. Se recomienda realizar estudios en nuestra población que permitan al sistema de salud en Panamá la implementación de estrategias que impacten sobre los factores de riesgo, dado que una buena parte de éstos son modificables.

Múltiples estudios han demostrado que variables externas como el nivel socioeconómico, el tipo de vivienda, el grado de hacinamiento, el acceso a agua potable y el nivel educativo del cuidador son factores de riesgo para el desarrollo de EDA. También han sido identificadas como factores de riesgo variables inherentes al paciente, como el estado de malnutrición, sumado a la deficiencia de zinc y la presencia de anemia.

La lactancia materna exclusiva por al menos cuatro meses constituye un factor protector importante contra el desarrollo de enfermedad diarreica.

Gracias a los esfuerzos de la OMS, en colaboración con las autoridades sanitarias panameñas, con respecto a la promoción de la importancia de la lactancia materna exclusiva, la educación sanitaria, la incorporación de la vacuna del rotavirus al programa ampliado de inmunización, aunado al desarrollo de las sales de rehidratación oral, se ha logrado una disminución de la morbimortalidad; sin embargo, aún hacen falta medidas más enérgicas que contribuyan a lograr una mayor disminución de los casos de diarrea en la población infantil en Panamá 
REFERENCIAS BIBLIOGRÁFICAS

[1] Herrera Benavente I, Comas García A, Mascareñas de los Santos A. Impacto de las enfermedades diarreicas agudas en América Latina. Justificación del establecimiento de un Comité de Enfermedades Diarreicas en SLIPE. Revista Latinoamericana de Infectología Pediátrica [Internet]. 2018 [citado el 23 de mayo 2020];31(1):8-16. Disponible en: https://www.medigraphic.com/pdfs/infectologia/lip2018/lip181c.pdf

[2] Enfermedades diarreicas [Internet]. Organización mundial de la salud. 2017 [citado el 11 de Noviembre de 2019]. Disponible en:

http://www.who.int/mediacentre/factsheets/fs330/es/

[3] Reyes U, Reyes K, Santos L, Luévanos A, Guerreros M, Martínez $P$ et al. Enfermedad diarreica aguda en niños. Salud Quintana Roo [Internet]. 2018 [citado el 23 de Mayo de 2020]; 11(40):34-41. Disponible en:

https://salud.qroo.gob.mx/revista/index.php/component/ content/article id=

[4] Mamo, Hailu A. Assessment of Prevalence and Related Factors of Diarrheal Diseases among Under-Five Year's Children in Debrebirehan Referral Hospital, Debrebirehan Town, North Shoa Zone, Amhara Region, Ethiopia. Open Access Library Journal [Internet]. 2014 [citado el 14 de diciembre del 2019]; 1:283. Disponible en:

http://dx.doi.org/10.4236/oalib.1100283

[5] Ministerio de Salud de Panamá. Análisis de Situación de Salud [Internet]. Panamá: Ministerio de Salud de Panamá; 2017 [citado 11 de abril de 2020]. Disponible en: http://www.minsa.gob.pa/sites/default/files/publicacione s/asis 2015.pdf

[6] Ministerio de Salud de Panamá. Indicadores de Salud Básicos [Internet]. Panamá: Ministerio de Salud de Panamá; 2017 [citado 12 de abril de 2020]. Disponible en: http://www.minsa.gob.pa/sites/default/files/publicaciongeneral/indicadores basicos de salud 2017 .pdf

[7] Ministerio de Salud de Panamá. Boletín Estadístico-Anuario 2017 [Internet]. 2018 [citado el 2 de febrero del 2020]. Disponible en:

http://www.minsa.gob.pa/sites/default/files/publicaciongeneral/boletin 2017.pdf

[8] Contraloría de la República de Panamá. Encuesta de indicadores múltiples por conglomerados 2013 [Internet]. Panamá: Contraloría de la República de Panamá; 2014 [citado 11 de abril de 2020]. Disponible en:

https://www.contraloria.gob.pa/inec/archivos/MICS FINA L.pdf
[9] SinmegnT, Asres G, Shimeka A. Determinants of childhood diarrhea among underfive children in Benishangul Gumuz Regional State, North West Ethiopia. BMC Pediatrics [Internet]. 2014 [citado el 5 de marzo de 2020]; 14:102. Disponible en:

http://www.ncbi.nlm.nih.gov/pubmed/24731601

[10] Godana W, Mengistie B. Determinants of acute diarrhoea among children under five years of age in Derashe District, Southern Ethiopia. Rural and RemoteHealth [Internet]; 2013 [citado el 8 de marzo de 2020]; 13: 2329. Disponible en:

http://www.rrh.org.au/publishedarticles/article_print 23 29.pdf

[11] Alparo Herrera Indhira, Fabiani Hurtado Nelly Rocío, Espejo Herrera Nadia. Factores de riesgo para enfermedad diarreica aguda con deshidratación grave en pacientes de 2 meses a 5 años. Rev. bol. ped. [Internet]. 2014 [citado el 9 de junio de 2019]; 53(2): 65-70. Disponible en: http://www.scielo.org.bo/scielo.php?script=sci_arttext\&p id=S1024-06752014000200002\&lng=es

[12] Ganguly E, Sharma P, Bunker C. Prevalence and risk factors of diarrhea morbidity among underfive children in India: A systematic review and meta-analysis [Internet]. India: HHS Public Access; 2016 [citado el 10 June de 2020]. Disponible en:

https://doi.org/10.32677/IJCH.2015.v02.i04.004

[13] Huffman SC, Combest C. Role of breast-feeding in the prevention and treatment of diarrhoea. J DiarrhoealDis Res [Internet]. 1990 [citado el 8 de abril de 2019]; 8(3):68-81. Disponible en: http://www.ncbi.nlm.nih.gov/pubmed/2243179

[14] Mariños C, Uchya J, Medina J. Determinantes sociales de la enfermedad diarreica aguda, como identificar el riesgo y la protección para la intervención sanitaria multivalente en los niños menores de tres años en el Perú. Revista Peruana de Epidemiología [Internet]. 2014 [citado 10 de abril de 2020]; 18(1):1-8. Disponible en:

http://www.redalyc.org:9081/articulo.oa?id=2031326770 05.

[15] Jill W, Wenjing T, Jenny L, Forsberg B. Diarrheal Diseases in Low- and Middle-Income Countries. The Open InfectiousDiseasesJournal [Internet]. 2010 [citado el 12 de abril 2020]; 4:113-124. Disponible en: http://dx.doi.org/10.2174/1874279301004010113

[16] Lamberti L, Fischer Walker C, Noiman A, Victora C, Black R. Breastfeeding and the risk for diarrhea morbidity and mortality. BMC PublicHealth [Internet]. 2011 [citado el 14 de Julio de 2020];11(Suppl 3): S15. Disponible en: 
https://www.ncbi.nlm.nih.gov/pmc/articles/PMC3231888

L

[17] Farthing M, Salam M, Lindberg G, Dite P, Khalif I, SalazarLindo $E$ et al. Diarrea aguda en adultos y niños: una perspectiva mundial. [Internet]. EE UU: Organización Mundial de Gastroenterología; 2012 [Citado el 14 de diciembre del 2019]. Disponible en:

http://www.worldgastroenterology.org/UserFiles/file/gui delines/acute-diarrhea-spanish-2012.pdf.

[18] Schilling K, Mintz E, Rothenberg R, Bopp C, Levine M, Nataro $J$ et al. Factors Associated with the Duration of Moderate-to-Severe Diarrhea among Children in Rural Western Kenya Enrolled in the Global Enteric Multicenter Study, 2008-2012. [Internet] The American Journal of Tropical Medicine and Hygiene. 2017 [citado el 10 de junio de 2020];97(1):248-258. Disponible en:

https://www.ncbi.nlm.nih.gov/pmc/articles/PMC5508904 \#: : :text=From\%20previous\%20research\%2C\%20promine nt\%20risk,antimicrobial\%20use\%2C14\%E2\%80\%9316\%20 poor

[19] Instituto Conmemorativo Gorgas de Estudios de la Salud. Estudio de los conocimientos, actitudes y prácticas, en lactancia materna, del personal de salud del primer nivel de atención del Ministerio de Salud y de la Caja del Seguro Social, en las Regiones Metropolitana de Salud y San Miguelito, año 2011. Panamá; 2012. [citado el 7 de junio de 2020] Disponible en:

http://www.gorgas.gob.pa/images/lactancia/ESTUDIO\%2 OCAP\%20LACTANCIA\%20MATERNA.pdf

[20] Guía para la atención integrada de niños y niñas desde el nacimiento hasta los 9 años de edad [Internet]. Ministerio de Salud - Caja del Seguro Social.2013. [citado el 7 de junio del 2020]. Disponible:

http://www.minsa.gob.pa/sites/default/files/normativida d/guia_integral 0.pd

[21] Ferreira E, Mongua N, Diaz J, Delgado g, Baez R, Cruz L et al. Diarreas agudas y prácticas de alimentación en niños menores de cinco años en México. Salud Pública de México [Internet]. 2013 [citado el 17 de abril 2020]; 55(2): 314-322. Disponible en:

http://www.scielosp.org/scielo.php?pid=S003636342013000800031\&script=sci abstract\&tIng=es

[22] Rodríguez, F. D; Borace, R; A, López; Cuellar M; Instituto Conmemorativo Gorgas de Estudios de la Salud; UNICEF Relación Costo Beneficio de las intervenciones de promoción sobre lactancia materna vs. laboratorios de fórmulas lácteas. Panamá. [citado 7 de junio de 2020] Disponible en: www.gorgas.gob.pa/wpcontent/uploads/2014/08/LACTANCIA-COSTOBENIFICIO.pdf

[23] Encuesta Nacional de Salud Sexual y Reproductiva (ENASSER 2009). Instituto Conmemorativo Gorgas. Panamá. [citado 7 de junio de 2020] Disponible en: http://www.gorgas.gob.pa/aplicaciones/revistanoindexad as/pdf/3\%20Enasser\%202009-Informe\%20Final.pdf

[24] Gobierno Federal. Atención, diagnóstico y tratamiento de Diarrea aguda en adultos en el primer nivel de atención [Internet]. México: Gobierno Federal [citado 11 de noviembre de 2019]. Disponible en:

http://www.cenetec.salud.gob.mx/descargas/gpc/Catalog oMaestro/106 GPC Diarreaagudaadultos/SSA $10608 \mathrm{G}$ RR.pdf

[25] NG J, López O, González G. Impacto de la introducción de la vacuna contra el rotavirus en la hospitalización por gastroenteritis aguda grave en el Hospital del Niño de la Ciudad de Panamá. RevPanam Salud Pública [Internet]. [citado el 14 de abril del 2020]; 24(3): 189-194. Disponible en:

http://dx.doi.org/10.1590/S1020-49892008000900005

[26] Reyes-Gómez U, Reyes-Hernández KL, Santos-Calderón LA, et al. Enfermedad diarreica aguda en niños. Salud Quintana Roo. [Internet]. 2018 [citado el 7 de junio de 2020] ;11(40):34-41. Disponible en: https://www.medigraphic.com/cgibin/new/resumen.cgi?IDARTICULO=92483

[27] Radlovic N, Lekovic Z, Vuletic B, Radlovic V, Simic D. Acute diarrhea in children. Servian Archives of Medicine. [Internet]. 2015 [citado el 7 de junio del 2020];143(1112):755-762. Disponible en:

https://www.researchgate.net/publication/291529668 A cute Diarrhea in Children.

[28] Ullah Khan W, Sellen D. WHO | Zinc supplementation in the management of diarrhoea [Internet]. Who.int. 2011 [citado el 7 de junio del 2020]. Disponible en: https://www.who.int/elena/titles/bbc/zinc diarrhoea/en/ 